\title{
Quantitative aspects of chlamydial infection of the cervix
}

\author{
D HOBSON,* P KARAYIANNIS,* RUTH E BYNG,* ELISABETH REES, $†$ \\ I ANNE TAIT, $†$ AND JEAN A DAVIES † \\ From the Departments of *Medical Microbiology and +Venereology, University of Liverpool, Royal \\ Liverpool Hospital, Liverpool
}

SUMMARY In 580 women with Chlamydia trachomatis infection of the cervix, the degree of the infection was assessed by counting the number of chlamydial inclusions which developed in McCoy cell monolayers inoculated with cervical swab material under standardised conditions.

In $34 \%$ of these women inclusion counts were $<100$ per monolayer whereas in $36 \%$ the counts were $>1000$. Clinical features in each of these groups were compared to see if certain factors could be identified as the cause, or the result, of high-grade rather than low-grade infection.

A significant association was found between high inclusion counts and the presence of cervical mucopus or cervical ectopy. Oral contraceptives acted additively with ectopy but had no significant effect alone. Concurrent gonorrhoea did not affect the degree of chlamydial infection. High inclusion counts were more common in women under 20 years of age than in older women.

\section{Introduction}

Over the past five years, our combined clinical and laboratory investigations of women with genital tract infections with Chlamydia trachomatis have shown large variations between individual infected patients, both in the number and severity of clinical signs ${ }^{1}$ and in the number of chlamydial inclusions obtained from cervical swabs inoculated into McCoy cell tissue cultures. $^{2}$ In a group of 202 sexual contacts of men with nongonococcal urethritis (NGU), women who had cervical mucopus, or those who had cervical ectopy and were also using oral contraceptives, showed a significantly higher prevalence of chlamydial infection than did women without these clinical features. ${ }^{3}$ It seemed of interest to determine if these or other clinical factors might be correlated not only with a high rate of infection but with a more severe infection than in women in whom these factors were absent.

In McCoy cell tissue cultures infected with $C$ trachomatis only a single cycle of development of the agent occurs, and each intracellular inclusion found after incubation represents a single infective chlamydial particle in the original inoculum. Thus, when standard amounts of material from cervical

Address for reprints: Dr D Hobson, Department of Medical Microbiology, The Duncan Building, Royal Liverpool Hospital, Prescot Street, Liverpool L7 8XW

Received for publication 24 September 1979 swabs are inoculated and incubated under constant conditions in coverslip monolayers of McCoy cells the final total number of inclusions found over the whole cell sheet should give a direct quantitative measure of the number of infective chlamydial particles in the cervical secretions of each patient.

The sensitivity and reproducibility of the laboratory method we have used has been investigated previously; ${ }^{4}$ for example, in $48 \mathrm{McCoy}$ cultures inoculated with a single sample of $C$ trachomatis the counts on individual coverslips varied from the mean by a maximum of only $\pm 23 \%$, and, when a second swab was taken seven days after the first swab from 106 untreated infected women, the inclusion counts were of the same magnitude in both swabs in $92.5 \%$ of cases. Thus, the wide variations in inclusion counts we had observed between patients might be the result of differing degrees of infection in the cervical mucosa in individual patients.

The aim of this study was to see if certain clinical factors might be caused by, or be the cause of, highgrade rather than low-grade infection. In particular it seemed essential to consider (a) the physical state of the cervix - that is, whether or not mucopus or ectopy were present; (b) hormonal effects on cervical epithelium - that is, whether or not the patient was using oral contraceptives; (c) if chlamydial infection might be enhanced opportunistically by concurrent infections, especially gonorrhoea; and (d) the age of the patient. 
Accordingly, clinical and laboratory data were compared in the total of 580 women from whom $C$ trachomatis had been isolated in this laboratory between January 1975 and December 1978. This series excludes only women with postpartum infection and those who had recently received antibiotic treatment, but it was not restricted to women with a definite history of sexual contact with men with NGU and did not exclude women with concurrent gonorrhoea.

\section{Patients and methods}

\section{STUDY POPULATION}

The 580 women consecutively found to have chlamydial infection on laboratory examination were all patients attending the sexually transmitted disease (STD) clinics at the Liverpool Royal Infirmary or the Royal Liverpool Hospital or who had been referred to the Liverpool Women's Hospital from various sources because of suspected genital tract infections. Only women whose infections were first diagnosed in the puerperium, or who had been given chemotherapeutic agents within the previous four weeks, were excluded.

Clinically, the group consisted of 70 women from a consecutive series of sexual contacts of men with NGU, whose clinical findings have been compared with those of contacts without chlamydial infection, ${ }^{3}$ together with 49 infected women examined in a consecutive unselected series of patients attending a single STD clinic of the Royal Infirmary on specified days (Davies et al, unpublished data).

The remaining 341 women were not seen consecutively in the clinics; in fact, they had often been deliberately selected for laboratory investigation because they did show certain clinical signs of genital infection, or because they were contacts of men with NGU or with gonorrhoea, or for various other reasons which made it important to confirm or exclude chlamydial infection in their differential diagnoses and to assess its severity if present. This element of clinical selection does not affect the proposed correlation of laboratory findings with the clinical features presented by the patients, since the investigation is based on the laboratory's total number of chlamydia-positive specimens, unselected and completed in advance of the clinical data being known. Obviously, the proportion of women with such features as cervical mucopus, cervical ectopy, or concurrent gonorrhoea cannot be taken as comparable with that in clinically unselected patients seen consecutively in studies designed for clinicoepidemiological purposes. ${ }^{13}$

CLINICAL EXAMINATION

Examination of the patients' clinical signs was made and recorded in the standardised pattern described earlier. ${ }^{3}$

\section{COLLECTION OF SPECIMENS}

Swabs for chlamydial isolation were taken only by medical staff of the clinic experienced in the standardised technique previously described, ${ }^{5}$ which ensures that both the contents of the endocervical canal and the whole of any area of ectopic columnar epithelium present are sampled.

Swabs for Neisseria gonorrhoeae isolation and investigations for syphilis, trichomoniasis, or yeast infections were performed on all patients as previously described. ${ }^{3}$

\section{LABORATORY INVESTIGATIONS}

The procedures for the isolation of $C$ trachomatis and subsequent counts of the total number of chlamydial inclusions recovered have been previously described in detail. ${ }^{24-6}$ Briefly, particular attention was paid to the quantitative reproducibility of the isolation procedure-for example, immediate immersion of the swab into fluid transport medium, rapid transport to the laboratory and inoculation usually 2-4 hours after the swab was taken but occasionally after overnight storage at $4^{\circ} \mathrm{C}$, followed by inoculation of a standardised amount of homogenised swab material with centrifuge-assistance on to McCoy cell coverslip monolayers of constant age and cell density. For the first 260 swabs, untreated McCoy cells were used, but cells treated with cycloheximide were used for the final 200 specimens, and both methods were used simultaneously for the remaining 120 specimens. Cycloheximide treatment has been adopted because chlamydial inclusions can be identified more rapidly and easily than in fresh cells; the total number of inclusions recovered was consistently higher in treated cells, but rarely by more than twofold, so that the log frequency distribution of counts did not vary significantly between the two methods.

In all cases, the inclusion counts recorded represent the primary inoculation of the first cervical swab to be taken from each patient during her attendance in the clinic. All swabs were allocated serial laboratory numbers, and isolation and inclusion counting procedures were completed before the patient's identity and clinical features were reviewed in the laboratory.

\section{STATISTICAL METHOD}

The $\chi^{2}$ test was used for statistical comparison.

\section{Results}

INCLUSION COUNTS AND CLINICAL FEATURES The wide range of inclusion counts found on culture of the primary cervical swabs for the whole group of 
TABLE I Distribution of chlamydial inclusion counts and clinical factors in 580 women with C trachomatis infection of the cervix

\begin{tabular}{|c|c|c|c|c|c|c|}
\hline \multirow[b]{2}{*}{$i w c$} & \multirow[b]{2}{*}{ No of women } & \multicolumn{5}{|c|}{ \% of women* with positive clinical factor } \\
\hline & & Mucopus & Ectopy & $O C$ & Gonorrhoea & Aged $<20$ years \\
\hline $\begin{array}{r}<10 \\
10+ \\
100+ \\
1000+ \\
10000+\end{array}$ & $\begin{array}{r}84 \\
112 \\
178 \\
133 \\
73\end{array}$ & $\begin{array}{l}36 \\
36 \\
44 \\
69 \\
66\end{array}$ & $\begin{array}{l}58 \\
63 \\
67 \\
79 \\
75\end{array}$ & $\begin{array}{l}49 \\
50 \\
54 \\
62 \\
66\end{array}$ & $\begin{array}{l}38 \\
35 \\
44 \\
44 \\
34\end{array}$ & $\begin{array}{l}23 \\
24 \\
26 \\
35 \\
40\end{array}$ \\
\hline $\begin{array}{l}\text { Total No } \\
\text { of women }\end{array}$ & 580 & 290 & 402 & 306 & 232 & 168 \\
\hline
\end{tabular}

iwc $=$ No of inclusions per whole coverslip McCoy culture inoculated with cervical swab material

$\mathrm{OC}=$ Oral contraceptives

*In each inclusion-count group

580 women is shown in table I. Although the counts ranged from only one inclusion per whole coverslip (iwc) to $>75000$ they showed a substantially normal distribution with a median count of 450 inclusions; $476(85 \cdot 5 \%)$ of the swabs yielded $>10$ iwc and 207 $(36 \%)$ gave $>1000$ iwc.

The distribution of the main clinical factors is also outlined in table $I$. The overall incidence of mucopus, ectopy, and gonorrhoea was high, as was to be expected by the clinical selection of these women. Nevertheless, certain clinical factors were not uniformly distributed among the whole group.

The greater the inclusion count, the greater was the proportion of patients who were under 20 years of age, showed cervical ectopy or mucopus, or were taking oral contraceptives. There did not however seem to be any significant correlation between the height of the inclusion count and concurrent gonorrhoea.

These observations suggest that the degree or severity of infection in the cervical mucosa varied according to the presence or absence of certain clinical factors affecting the cervix. To evaluate their

TABLE II Distribution of clinical factors in relation to low or high inclusion counts

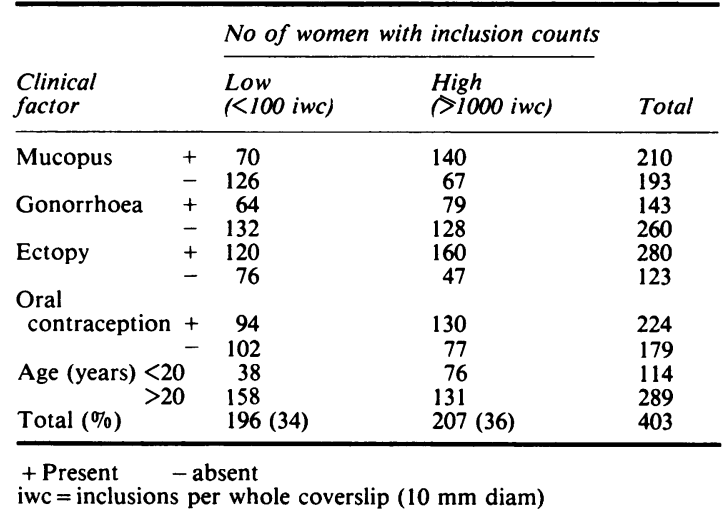

interactions more precisely the clinical features of women at the opposite ends of this frequency distribution of inclusion counts-that is, those with low counts of $<100$ iwc and those with high counts of $>1000$-were compared (table II).

\section{Cervical mucopus/concurrent gonorrhoea}

The amount of chlamydial growth in the cervical mucosa was significantly greater in women with mucopus than in those without (table II; $\chi_{1}^{2}=41 \cdot 1$, $\mathrm{P}<0.001$ ). A heavy chlamydial infection could be the actual cause of the mucopus but alternatively some other clinical factor could be the cause of both the mucopus and the high chlamydial count. For the latter reason, the fact that $143(35 \%)$ of the 403 women had gonorrhoea concurrently with their chlamydial infection had to be taken into account. Gonorrhoea itself is obviously a common cause of cervical mucopus and chlamydial infection might possibly be enhanced opportunistically in tissues already being damaged by $N$ gonorrhoeae.

However, high chlamydial counts were not significantly more frequent in women with associated gonorrhoea than in women with chlamydial infection alone $\left(\chi_{1}^{2}=1 \cdot 33, \mathrm{P}>0 \cdot 1\right)$ (table II). Furthermore, the

TABLE III Prevalence of cervical mucopus in relation to inclusion counts in women with or without concurrent gonorrhoea

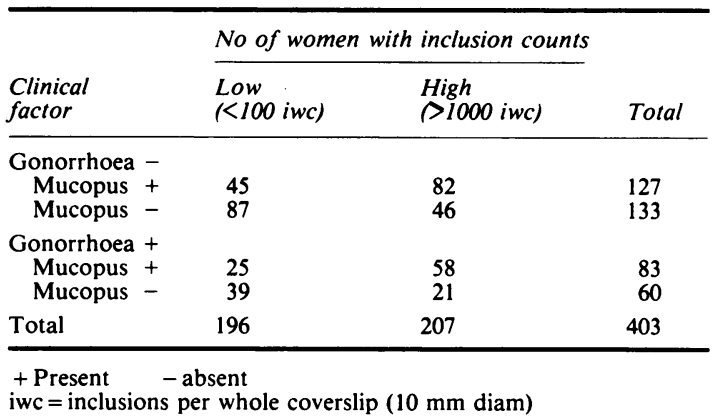


association between mucopus and high chlamydial counts was not dependent on a concomitant infection with $N$ gonorrhoeae, as was shown by evaluating separately the data in the groups of women with and without gonorrhoea (table III).

Mucopus was present in a high proportion of all the women studied $(210 / 403,52 \%)$; this could be partly due to the clinical selection of the cases before referral for laboratory diagnosis. However, the proportion of cases with mucopus was similar in women with chlamydial infection alone $(127 / 250,65 \%)$ and in chlamydia-positive women with concurrent gonorrhoea $(83 / 143,70 \%)\left(\chi_{1}^{2}=3 \cdot 13, \mathrm{p}>0 \cdot 05\right)$. Moreover, there was a significant association between the presence of mucopus and high inclusion counts whether or not the woman had a chlamydial infection alone $\left(\chi_{1}^{2}=23 \cdot 36, \mathrm{P}<0 \cdot 001\right)$ or concomitant gonorrhoea $\left(\chi_{1}^{2}=17 \cdot 14, \mathrm{P}<0 \cdot 001\right)$.

\section{Cervical ectopy/oral contraceptives}

When these clinical factors were considered separately (table II) each showed a significant association with the height of the inclusion count. There was little difference between simple ectopy or hypertrophic ectopy in relation to inclusion counts and both have been combined for statistical analysis. Because of the clinical selection cervical ectopy was present in over two-thirds of the $\mathbf{4 0 3}$ women studied (table II). However, $57 \%$ of these women had high inclusion counts compared with $38 \%$ of women without ectopy $\left(\chi_{1}^{2}=12 \cdot 3, \quad P<0 \cdot 001\right)$. Oral contraceptives were being used by $56 \%$ of the 403 women and $58 \%$ of these patients had high inclusion counts compared with $43 \%$ of women not taking oral contraceptives $\left(\chi_{1}^{2}=8 \cdot 98, \mathrm{P}<0 \cdot 01\right)$.

However, cervical ectopy and oral contraceptives were obviously not independent variables, since only $106(38 \%)$ of the 280 women with ectopy were not taking oral contraceptives and only $50(22 \%)$ women using oral contraceptives did not have cervical ectopy. In fact, the association between the use of oral contraceptives and the presence of ectopy was highly significant $\left(\chi_{1}^{2}=47.08, \mathrm{P}<0.001\right)$; thus these two clinical factors probably interact in their effect on the height of the inclusion count, as they have previously been shown to affect the incidence of chlamydial infection. ${ }^{3}$ To assess this interaction, the four possible combinations of the two factors were further analysed (table IV):

1. In patients without cervical ectopy, high counts were found in $32 \%$ of women not taking, and in $48 \%$ of women taking, oral contraceptives. The effect of oral contraceptives alone was not significant $\left(\chi_{i}^{2}=3 \cdot 42, \mathrm{P}>0 \cdot 05\right)$.

2. In patients with cervical ectopy, high counts were found in $51 \%$ of women not taking, and in $61 \%$ of women taking, oral contraceptives. The effect of oral contraceptives alone was not significant $\left(\chi_{1}^{2}=2 \cdot 67, \mathrm{P}>0 \cdot 1\right)$.

3. In patients with oral contraceptives, high counts were found in $48 \%$ of women without ectopy and in $61 \%$ of women with ectopy. The effect of ectopy alone in these women was not significant $\left(\chi_{1}^{2}=2 \cdot 66, \mathrm{P}>0 \cdot 1\right)$.

4. In patients without oral contraceptives, high counts were found in $32 \%$ of those without ectopy and in $51 \%$ of those with ectopy. This effect of ectopy alone was significant $\left(\chi_{1}^{2}=6 \cdot 67\right.$, $P=0 \cdot 01$ ).

When the patients with neither ectopy nor oral contraceptives were compared with those with both ectopy and oral contraceptives, the difference in the proportion of cases with high inclusion counts, $32 \%$ and $61 \%$ respectively, was significant $\left(\chi_{1}^{2}=17 \cdot 8\right.$, $\mathrm{P}<0.001)$.

These results suggest that ectopy and oral contraceptives acting jointly are likely to be associated with high chlamydial counts and that ectopy alone can result in high counts even if the ectopy has not been produced by, or the cervical mucosa has not been acted upon by, oral contraceptives. The effect of oral contraceptives on chlamydial counts appears to be indirect by way of its capacity to produce ectopy. When these possible effects are discounted, by dividing the women into groups (table IV), oral contraceptives appear to have no significant direct effect on the degree of chlamydial infection.

TABLE IV Interaction of cervical ectopy, oral contraceptives, and inclusion counts

\begin{tabular}{|c|c|c|c|c|c|}
\hline \multicolumn{2}{|c|}{ Clinical factors } & \multicolumn{3}{|c|}{ No of women with inclusion count } & \multirow[b]{2}{*}{$\begin{array}{l}\% \text { with high } \\
\text { count }\end{array}$} \\
\hline Ectopy & $O C$ & $\begin{array}{l}\text { Low } \\
\text { (<100 iwc) }\end{array}$ & $\begin{array}{l}\text { High } \\
\text { S } 1000 \text { iwc) }\end{array}$ & Total & \\
\hline $\begin{array}{l}- \\
- \\
+ \\
+ \\
\text { Total }\end{array}$ & $\begin{array}{l}- \\
+ \\
+ \\
+\end{array}$ & $\begin{array}{r}50 \\
26 \\
52 \\
68 \\
196\end{array}$ & $\begin{array}{r}23 \\
24 \\
54 \\
106 \\
207\end{array}$ & $\begin{array}{r}73 \\
50 \\
106 \\
174 \\
403\end{array}$ & $\begin{array}{l}32 \\
48 \\
51 \\
61\end{array}$ \\
\hline
\end{tabular}

+ Present - absent

iwc $=$ inclusions per whole coverslip $(10 \mathrm{~mm}$ diam $)$

OC $=$ Oral contraceptives 


\section{Age factor}

Of the total of 403 women with chlamydial infections, $114(28 \%)$ were under 20 years of age (table II); of these, $76(67 \%)$ had high inclusion counts compared with $131(45 \%)$ of the 289 women over 20 years of age $\left(\chi_{1}^{2}=14 \cdot 9, \mathrm{P}<0.001\right)$.

Despite this significant difference in the degree of infection between the under-20 age group and older women, no significant differences emerged in any of the other clinical features (table V).

\section{Other clinical features}

There were 119 women with one or more previous children and 22 pregnant women in the group examined; 36 women had associated trichomonal infections, 89 were infected with yeasts, and 31 had signs or symptoms suggesting salpingitis. There was no evidence that the frequency of high chlamydial counts was greater in these women than in women without these clinical features. However, the small numbers limit conclusive statistical analysis.

No significant difference was found in the proportion of women with high inclusion counts in successive weeks of the menstrual cycle. In the group of 403 women studied, 22 were pregnant, 32 had irregular periods or amenorrhoea, and in 20 the date of the last period was not recorded. In the $329(82 \%)$ women with a regular cycle of 26-30 days, the month was divided into four weeks starting from the first day of menstruation, and the cycle-week in which the primary cervical swab for $C$ trachomatis isolation was taken was recorded against the inclusion count obtained on culture (table VI).

\section{Discussion}

Current tissue culture methods for isolating $C$ trachomatis from cervical swabs of infected women provide a quantitative means of monitoring the reproducibility and sensitivity of the procedures themselves in continued diagnostic use ${ }^{24}$ and of detecting differences in the degree of infection between individual patients, since the number of inclusions which develop in McCoy cell culture is directly proportional to the number of viable chlamydial elementary bodies inoculated into the culture. $^{?}$

TABLE V Distribution of clinical features in relation to age

\begin{tabular}{lll}
\hline & \multicolumn{2}{l}{$\%$ of women } \\
\cline { 2 - 3 } Clinical feature & $<20$ years & $>20$ years \\
\hline Mucopus & 51 & 53 \\
Gonorrhoea & 40 & 39 \\
Ectopy & 75 & 68 \\
Oral contraceptives & 52 & 57 \\
\hline
\end{tabular}

In the present series of 580 women with chlamydial genital infections from whom a primary cervical swab was taken for laboratory culture before they had been given chemotherapeutic agents, the inclusion counts were normally distributed with a median value of 450 iwc, but the range of counts obtained was wide, with $73(13 \%)$ of the group having counts of $>10000$ iwc whereas $84(14 \%)$ had counts of $<10$ iwc. When the inclusion counts in the individual patients were related to the clinical features found at the time the swab was taken, the degree of infection (indicated by the inclusion count) apparently varied according to the presence or absence of cervical mucopus or ectopy, the use of oral contraceptives, and the age of the patient.

The increase in inclusion counts in women with cervical mucopus may be due to the following: (a) the greater the amount of secretion in the cervical canal the greater is the amount that might be collected on the swab, and hence the greater the number of elementary bodies available to infect the McCoy coverslip culture; (b) the presence of mucopus might non-specifically enhance the sensitivity of McCoy cells to chlamydial infection, resulting in a higher proportion of the potentially infective particles inoculated successfully forming inclusions in McCoy cells; (c) mucopus may be merely an indicator of another concurrent infection, especially gonorrhoea, which might be capable of enhancing the chlamydial infection-that is, $C$ trachomatis might thus show the characteristics of a secondary opportunistic invader; and (d) mucopus might in fact be the direct result of chlamydial infection and thus occur most frequently in those with the greater degree of infection, as indicated by the inclusion count.

In our experience the thick tenacious nature of the mucopus often results in rather less than more cervical secretion being obtained per swab and limits the amount of homogenisation of material from the swab before tissue culture inoculation. Suspensions of cervical mucopus from patients with STDs but without current chlamydial infection are slightly but consistently inhibitory to the growth of laboratory strains of $C$ trachomatis in McCoy cultures (Hobson and Lee, unpublished data).

\section{TABLE VI Inclusion counts in relation to menstrual cycle}

\begin{tabular}{lllllll}
\hline & \multicolumn{3}{c}{ Week of cycle* } & \\
\cline { 2 - 6 } & 1 & 2 & 3 & 4 & Total \\
\hline No of patients & 60 & 112 & 79 & 78 & 329 \\
No with high count $(>1000$ iwc) $)$ & 31 & 60 & 40 & 42 & 173 \\
\% with high count & 52 & 54 & 51 & 54 & 53 \\
\hline * At the time of primary cervical swab & & & & \\
†Inclusion count in primary cervical swab & & &
\end{tabular}


Concurrent infection with trichomonads, yeasts, or $N$ gonorrhoeae appeared to have no effect on the degree of chlamydial infection. It is interesting however that women with gonorrhoea have previously been found 89 to have a higher rate of chlamydial cervical infection than have contacts of men with NGU or attenders of STD clinics without gonorrhoea. This incidence may be increased because coincidental infections in the more promiscuous STD clinic patients, who must frequently be exposed to both types of infective agent, are highly likely rather than because gonorrhoea predisposes to or stimulates a previously latent or low-grade chlamydial infection. Furthermore, women who had concomitant infections did not develop mucopus more frequently than did women with chlamydial infections alone; in both groups the presence of mucopus was associated with high chlamydial inclusion counts. These results thus suggest that the presence of mucopus in a woman with gonorrhoea may, in certain cases, be the result of an associated chlamydial infection rather than due to the gonococcal infection alone.

There is a highly significant association between the chlamydial isolation rate and the incidence of cervicitis in contacts of men with NGU, ${ }^{310}$ and treatment of the chlamydial infection is quickly followed by disappearance of mucopus. ${ }^{3}$ The present results support and extend these findings-that is, $C$ trachomatis can cause endocervicitis and the greater the degree of infection the more likely are the signs of endocervicitis to be present.

In contacts of men with NGU seen in Liverpool ${ }^{3}$ a higher rate of chlamydial infection was found when both cervical ectopy and contraceptive steroids were present than when either occurred alone or when neither was present. All the infected women from this smaller study group ${ }^{3}$ are included in the present larger and more varied series, which shows that not only the incidence but also the degree of chlamydial infection was greater in women with ectopy and contraceptive steroids than in women with neither. Oral contraceptives alone did not appear to have any influence on the degree of infection but, in contrast, the inclusion counts were significantly higher in those with ectopy alone-even in the absence of oral contraceptives-than in women without ectopy. Since the site of the squamo-epithelial junction is determined during fetal life, ${ }^{11}$ infection with $C$ trachomatis cannot be a cause of cervical ectopy, although infection may convert a small area of simple ectopy to a larger area of hypertrophic ectopy as a result of oedema and congestion. ${ }^{3}$

The most likely explanation of the significant association between the presence of ectopy and both an increased rate of infection ${ }^{3}$ and an increase in the degree of that infection demonstrable in tissue culture is simply that the greater the area of columnar epithelium exposed on the vaginal aspect of the cervix the greater is the chance that infection will be acquired after coitus with an infected man, the greater is the number of susceptible columnar cells available for progressive growth cycles of the infective agent, and the greater is the area that can be sampled by taking swabs.

The role of oral contraceptives is more difficult to determine and is almost certainly multifactorial. The frequency and size of cervical ectopy is itself increased by the use of contraceptive steroids, ${ }^{12}{ }^{13}$ and thus the chlamydial inclusion count is likely to be significantly increased if oral contraceptives are considered overall, but much of this increase may be the result of the ectopy associated with the use of oral contraceptives and not uniquely due to the drugs. However, oral contraceptives may possibly also have direct hormonal effects on columnar epithelium in vivo which increases the chance that a highly productive infection with $C$ trachomatis will develop. Contraceptive steroids have a progestational effect, producing a large outflow of alkaline mucus from the endocervical glands. ${ }^{12}$ The addition of steroids to McCoy cell cultures increases their susceptibility to infection with laboratory strains of $C$ trachomatis, resulting in an increase in the number of infective progeny produced by the inoculation of a given number of elementary bodies. ${ }^{14}$ Thus, as suggested by the present findings, the hormonal effects of oral contraceptives would be seen most clearly when acting in conjunction with cervical ectopy, especially since the action of these steroids increases the bulk of the cervix and produces eversion. ${ }^{12}$ However, in the present series of women there was no significant association between the height of the inclusion count and other hormonal influences, either in the normal menstrual cycle or in pregnancy, although a much larger series of women would need to be examined to confirm the latter.

A further feature needing more extensive investigation is the highly significant association between the age of the patient and the degree of her infection in the present series of women. This association might possibly reflect anatomical and physiological, including hormonal, differences between women under 20 years of age and older women, but the present findings do not suggest that the increased inclusion counts are the results of a greater use of oral contraceptives or of a greater frequency of cervical ectopy than in women between 21-45 years of age. However, the exact area of ectopy was not measured in these women. The size of cervical ectopy diminishes with age owing to metaplasia, whereby columnar epithelium is gradually replaced by 
squamous epithelium, and this process is often initiated at puberty or in the first pregnancy. ${ }^{11}$ Thus, possibly the area of ectopic columnar cells, rather than the frequency of ectopy, may vary between young and older women to influence their differing degrees of chlamydial infection.

It is also possible that this age difference may have an immunological basis. In girls aged between 14-20 years a greater proportion of the infections are probably the first chlamydial infections to which they have been exposed than is the case in older women with a longer period of sexual activity. Thus, the replication of $C$ trachomatis may be less likely to be brought quickly under control by humoral or cervical antibody or by cell-mediated immune responses in young women than in older women with a greater chance of previous immunological experience. Humoral antibody against $\boldsymbol{C}$ trachomatis is frequent and persistent in women attending STD clinics and its frequency increases with age. ${ }^{15-17}$ Although it is not yet clear that antibody acquired by previous experience will prevent infection, the possibility that it might modify the degree of infection and hence the clinical signs associated with high-inclusion count infection merits further study.

This work was supported by a project grant from the Medical Research Council.

\section{References}

1. Rees E, Tait IA, Hobson D, Johnson FWA. Chlamydia in relation to cervical infection and pelvic inflammatory disease. In: Hobson D, Holmes KK, eds. Nongonococcal Urethritis and Related Infections. Washington DC: American Society for Microbiology, 1977:67-76.

2. Johnson FWA, Hobson D, Rees E, Tait IA Quantitative aspects of the growth of Chlamydia trachomatis in diagnostic tissue culture procedures. In: Hobson D, Holmes KK, eds. Nongonococcal Urethritis and Related Infections. Washington DC: American Society for Microbiology, 1977:309-13.
3. Tait IA, Rees E, Hobson D, Byng RE, Tweedie MCK. Chlamydial infection of the cervix in contacts of men with nongonococcal urethritis. Br J Vener Dis 1980;56:37-45.

4. Johnson FWA, Hobson D. Factors affecting the sensitivity of replicating McCoy cells in the isolation and growth of Chlamydia A (TRIC agents). J Hyg Camb 1976; 76: 441-51.

5. Davies JA, Rees E, Hobson D, Karayiannis P. Isolation of Chlamydia trachomatis from Bartholin's ducts. Br J Vener Dis 1978; 54:409-13.

6. Johnson FWA, Chancerelle LYJ, Hobson D. An improved method for demonstrating the growth of chlamydiae in tissue culture. Med Lab Sci 1978;35:67-74.

7. Gordon FB, Dressler HR, Quan AL, Thomas JJ. Effect of ionizing irradiation on susceptibility of McCoy cell cultures to Chlamydia trachomatis. Appl Microbiol 1972; 23:123-9.

8. Hobson D, Rees E. The role of Chlamydia trachomatis in female genital infections and in conjunctivitis of the newborn. In: Brumfitt W, ed. New Perspectives in Clinical Microbiology. London: Kluwer Medical, 1978: 109-23.

9. Hilton AL, Richmond SJ, Milne JD, Hindley F, Clarke SKR Chlamydia $\mathrm{A}$ in the female genital tract. Br J Vener Dis 1974;50: $1-10$

10. Dunlop EMC, Al-Husseini MK, Freedman A, et al. Infection by TRIC agent and other members of the Bedsonia group; genital infection and disease of the eye. Trans Ophthalmol Soc UK 1966;86: 321-34.

11. Singer A, Jordan JA. The anatomy of the cervix. In: Jordan JA, Singer A, eds. The Cervix. London: WB Saunders, 1976: 13-36

12. Jordan JA, Singer A. Effect of oral contraceptive steroids upon epithelium and mucus. In: Jordan JA, Singer A, eds. The Cervix. London: WB Saunders, 1976: 192-207.

13. Goldacre MJ, Loudon N, Watt B, et al. Epidemiology and clinical significance of cervical erosion in women attending a family planning clinic. $B r$ Med $J$ 1978; 1:748-50.

14. Bushell AC, Hobson D. Effect of cortisol on the growth of $C$ trachomatis in McCoy cells. Infect Immun 1978; 71:946-53.

15. Richmond SJ, Caul EO. Single-antigen indirect immunofluorescence test for screening venereal disease clinic populations for chlamydial antibodies. In: Hobson D, Holmes $\mathrm{KK}$, eds. Nongonococcal Urethritis and Related Infections. Washington DC: American Society for Microbiology, 1977:259-65.

16. Treharne JD, Dines RJ, Darougar S. Serological responses to chlamydial ocular and genital infections in the United Kingdom and Middle East. In: Hobson D, Holmes KK, eds. Nongonococcal Urethritis and Related Infections. Washington DC: American Society for Microbiology, 1977:249-58.

17. Schachter J, Dawson CR. Comparative efficiency of various diagnostic methods for chlamydial infection. In: Hobson D, Holmes KK, eds. Nongonococcal Urethritis and Related Infections. Washington DC: American Society for Microbiology, 1977:336-41. 
Antibiotics and Chemotherapy: Current Topics. Edited by R N Grüneberg, 1980. Pp 219. MTP Press, Lancaster. Price £12.95.

It is difficult for most people to keep abreast of the rapidly changing field of antibiotics and chemotherapy. In this book eight topics have been chosen for review which are of general interest. These include a very lucid exposition of the treatment of infective endocarditis, the vexed question of the prophylactic use of antibiotics, the treatment of anaerobic infections, and the use of combinations of antibiotics, as well as an exhaustive review of the cephalosporins, antibiotic policies, and the treatment of gonorrhoea and non-specific genital infection. Venereologists are fortunate in that most of the conditions they commonly deal with respond well to antibiotics, although they need to be aware of changing patterns of sensitivity. particularly of the gonococcus.

The chapter on gonorrhoea and nonspecific genital infection is written from a practical standpoint and gives a good description of current practice with references to the recent literature up to 1978 . The problems posed by the emergence of $\beta$-lactamase-producing strains of gonococci are discussed rather briefly; only spectinomycin and cefuroxime are mentioned as alternative methods of treating infections with these strains. Recent work in the field of non-specific genital infection is described and due emphasis placed on those infections not due to chlamydia for which, in the absence of defined pathogens, treatment still has to be empirical.

These articles provide good reviews of current ideas in a number of important fields and are all backed up by extensive references. The book is well produced with a good index; it is not unduly expensive by today's standards. It can be recommended as a clearly written description of some important applications of antibiotics and their rational use.

A E Wilkinson

Fortschrifte der Praktischen Dermatologie und Venerologie (Advances in Dermatology and Venereology). Edited by $O$ BraunFalco and H H Wolff, 1979. Pp 396. Springer Verlag, Berlin. Price DM 98, US\$54.90.

This volume is somewhat unbalanced, dealing with venereology in 40 pages out of a total of 385 . Sections on gonorrhoea and syphilis are mostly confined to the German literature and the penicillin schedules suggested for late syphilis strike one as complex and fussy and include even penicillin infusions. The recent specific immunoglobulin serological tests may point the way to having tests of activity but some doubts on this score still remain.
It is a paradox that while in all Western European countries the relative number of women with gonorrhoea has gone up-approaching that of men-the ratio in West Germany has remained firmly static with three times as many men as women having been diagnosed as being infected.

The number of patients infected with $\beta$-lactamase-producing gonococci has remained very low and no routine test to determine the incidence of $\beta$-lactamase producers is at present suggested. Systemic gonorrhoea is very well described and illustrated.

In the section dealing with chlamydiaassociated urethritis it is good to see that proper weight has been given to the pioneering work of Lindner, a Viennese ophthalmologist, who laid the foundation to subsequent work in this sphere in amazing detail as early as 1908 and 1910 .

The long section on chancroid, which is rare in Germany, might appear out of proportion when it is realised that no mention is being made of such important and common conditions as genital herpes, sexually transmitted hepatitis, Reiter's disease, or specific vaginitis and its complications.

All in all, the section on venereal diseases will have little appeal for venereologists and probably give too little practical detail to be of much value to the dermatologist who also dabbles in venereology.

GW Csonka

\section{Notice}

\section{Symposium on trichomoniasis}

An international symposium on trichomoniasis, organised by the Commission on Trichomoniasis of the General Council of the Polish Parasitological Society, will take place between 13 and 15 July 1981 in the Medical Academy in Białystok as a satellite meeting to the VIIth International Congress of Protozoology (Warsaw, 5-11 July 1981).

The main topics will be: biology of Trichomonas vaginalis Donné, including culture; biochemistry and physiology; immunology and serology; clinical and experimental pathology; epidemiology; diagnosis; and treatment and prophylaxis.
Those interested in attending the meeting are asked to apply by 1 January 1981 to either Professor S Soszka (President of the organising committee), Institute of Gynaecology and Obstetrics, SkYodowska Curie Str 24A, 15-097 Białystok, Poland, or Professor B Honigberg (Vice-president), Department of Zoology, University of Massachusetts, Amhert, Massachusetts 01003, USA, or Professor M Müller (vice president), Rockefeller University, New York, NY 10021, USA.

The papers will be published in English in the periodical Wiadomości Parazytologiczne after being read at the symposium.

\section{Correction}

In the paper by Hobson et al on "Quantitative aspects of chlamydial infection of the cervix" $(1980 ; 56: 156-62)$ the results in the second paragraph on page 156 should be as follows: the proportion of women with mucopus with chlamydial infection alone was $127 / 260(48 \cdot 8 \%)$ and not $127 / 250(65 \%)$ and that of chlamydiapositive women with gonorrhoea was $83 / 143(58 \%)$. 